\title{
A STUDY OF RIPPLE WAVE MOTION.
}

By F. R. Watson and W. A. Shewhart.

$\mathrm{W}$

AVE motion has been made the object of a large number of theoretical and experimental investigations to determine the physical properties of waves and also to explain the various phenomena of reflection, refraction, diffraction and interference. One of the most satisfactory experimental methods of attack has been found in the study of ripple waves, since in this case, we can visualize almost every phenomena of wave motion.

The object of this paper is to describe the development of a method by which ripple waves may be generated not only in steady patterns but also in patterns in which the waves apparently move very slowly. This allows a leisurely examination of the various phenomena. The method also allows the convenient exhibition of the waves to a lecture audience.

Historically, the investigation of ripples began in $\mathrm{I} 87 \mathrm{I}$ when Lord Kelvin ${ }^{1}$ observed that the propagation of ripples depended on surface tension. Matthieson ${ }^{2}$ tested the validity of Kelvin's formula, but, because of the rough measurements of the waves set up by a pin point piercing a jet of water, failed to obtain a great degree of accuracy. Ahrendt, ${ }^{3}$ Riess $^{4}$ and others made similar experiments, but it remained for Lord Rayleigh $^{5}$ to develop the first accurate method of investigation. To make visible the extremely small disturbances in the plane of the liquid surface, he used a modified form of Foucault's method of testing plane surfaces. Furthermore, he used the stroboscopic method for making the waves appear to stand still. Dorsey ${ }^{6}$ and Watson ${ }^{7}$ have extended and improved Rayleigh's method in making investigations on the surface tension of liquids.

Tyndall ${ }^{8}$ first made use of ripple waves to illustrate wave motion. Later, Vincen $t^{9}$ was able with more refined apparatus to obtain beautiful photographs of the same phenomena. H. Schultze ${ }^{10}$ devised an electrical

${ }_{1}^{1}$ Phil. Mag. (4), Vol. 42, p. 375, I87r.

${ }^{2}$ Wied. Ann., Vol. 38, p. II 8, I889.

${ }^{3}$ Exner's Rep. der Physik, Vol. 24, p. 318, I888.

${ }^{4}$ Exner's Rep. der Physik, Vol. 26, p. I02, I890.

${ }^{5}$ Lord Rayleigh's Collected Works, Vol. III., p. 383.

${ }^{6}$ Phys. Rev., Vol. 5, p. I73, 1897.

${ }^{7}$ Phys. Rev., Vol. 12, p. 257 , I90r.

${ }^{8}$ S. P. Thompson, "Light, Visible and Invisible," Chap. I.

${ }^{9}$ Phil. Mag., Vol. 43, p. 41 7; 45, I9r; 46, 290.

${ }^{10}$ Zeitsch. f. Instk., p. I5 I, 1907. 
method for producing ripples, modifications of which have been made by Pfund ${ }^{1}$ and Palmer. ${ }^{2}$ Waetzmann ${ }^{3}$ developed a method in which the waves were generated by intermittent puffs of air, and were made visible by flashes of light isoperiodic with the puffs. Waetzmann's method, with extensions and modifications, has been used in the present investigation.

Fig. $I$ is a diagram of the apparatus. Ripple waves were generated by puffs of air blown against the water surface in the glass-bottomed tank $A$. The puffs were secured by cutting a tube conveying compressed air and inserting in the gap a disc with a circular row of equally spaced holes. When the disc rotated, the current of air was periodically interrupted. The waves were made visible by the stroboscopic method. Light from an arc lamp was focused on the row of holes in the rotating disc, thus giving flashes isoperiodic with the puffs of air. By reflecting the light upward through the glass tank, a steady pattern of waves was revealed. Fig. 2 shows a photograph obtained with circular waves.

By using a second row of holes, which were fewer in number than

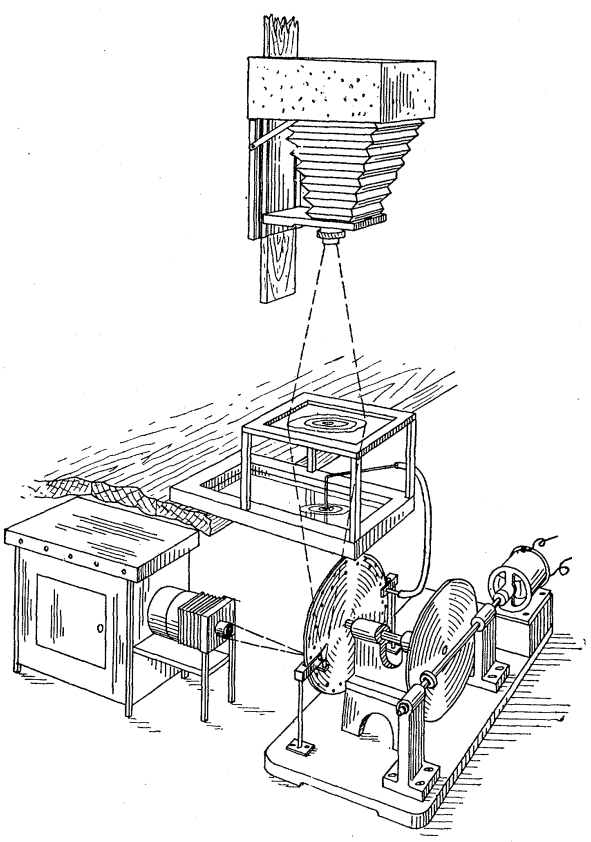

Fig. 1.

Diagram of apparatus showing how ripples are generated on a water surface by puff of air and made visible stroboscopically on the frosted glass plate above the water tank. those in the row already described, the flashes of light could be made to come a little slower than the puffs of air; the result being that the waves apparently moved forward slowly. This action allowed a leisurely study for the different phases of reflection, diffraction, etc., with slowly moving waves.

Trouble was experienced in getting steady patterns of waves, due to vibrations of the apparatus and fluctuations of the puffs of air. The vibrations were overcome by mounting the tank on a steady support. The fluctuations in the air puffs were almost entirely eliminated by using

1 Phys. Rev., Vol. 32, p. 324, I9II.

2 Phys. Rev., Vol. 33, p. 528, I9II.

${ }^{3}$ Physikalische Zeitschrift, Vol. I2, p. 866, I9II. 
a cast iron disc Io inches in diameter and one-fourth inch thick and facing it after it had been mounted on an axle so that it would run true. It was then mounted securely on a cast-iron base and rotated by a small wheel on an axle attached by a toggle joint to a I/6-H.P. direct-current motor. Variation of the speed of the disc was obtained by changing the resistance in series with the motor and also by shifting the position of contact of the small wheel with the disc.

To make the flashes of light sharp and definite in position, an arrangement of two small screens, each pierced by a small hole, was placed over the edge of the disc so that light could pass only during the instant that a hole in the disc came into coincidence with the holes in the screens. The reflecting mirror, which was small in area, was made by polishing the end of a circular aluminum rod cut at an angle of $45^{\circ}$ to its axis. The source of light was a carbon arc fed by a I Io-volt direct current. It gave good illumination except for the colors explained by Mrs. Ayrton. ${ }^{1}$ These proved to be troublesome when taking photographs. A pattern of waves that appeared well illuminated to the eye would show serious distortions on the photographic plate.

The position and form of the aperture for the puffs of air influenced the shape of the waves to a marked extent. Satisfactory results were obtained by using a small copper tube of about I mm. diameter placed near the water surface. It could easily be bent into any desired position.

The waves may be shown to an audience by mounting a mirror at an angle of $45^{\circ}$ over the surface of the tank so that the light is reflected to a screen. For this purpose it is desirable to let more light through so that the pattern will be well illuminated. This makes the definition of the waves less sharp but still satisfactory enough for demonstration.

Figs. 2 to 7 show some of the patterns investigated. Fig. 4 shows a curious overlapping interference. The patterns in Figs. 5, 6, and 7 were obtained by placing metal forms on the bottom of the glass tank and allowing the water surface to barely cover them. The metal must be free from oil or grease. In Fig. 6 the diffraction waves were made more intense by shielding the central portion from the camera for part of the exposure. The time of exposure varied from Io seconds to 20 minutes, depending on the amount of light. The length of the waves was measured to be nearly $0.4 \mathrm{~cm}$.

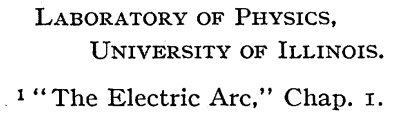




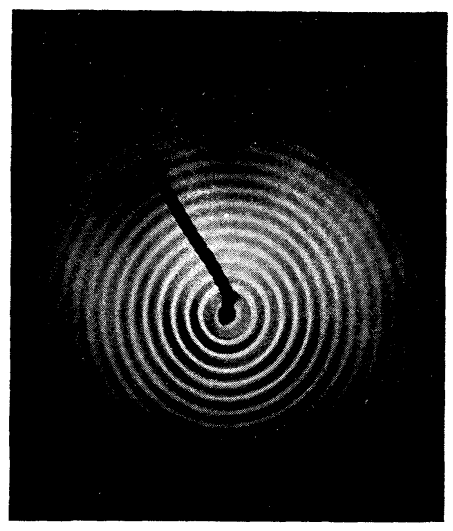

Fig. 2.

Circular waves.

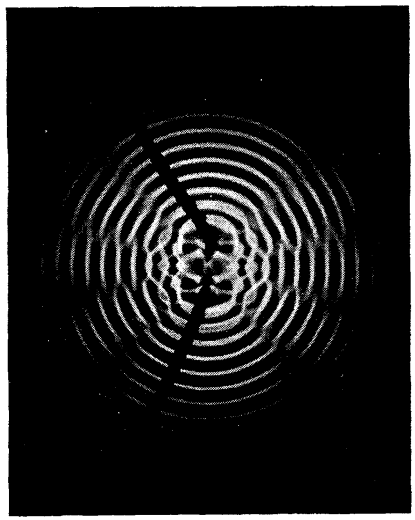

Fig. 4.

Interference of two sets of circular waves with sources close together.

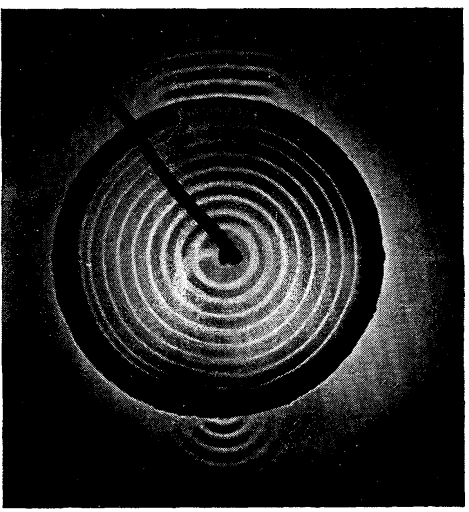

Fig. 6.

Diffraction of waves through narrow and wide aperture.

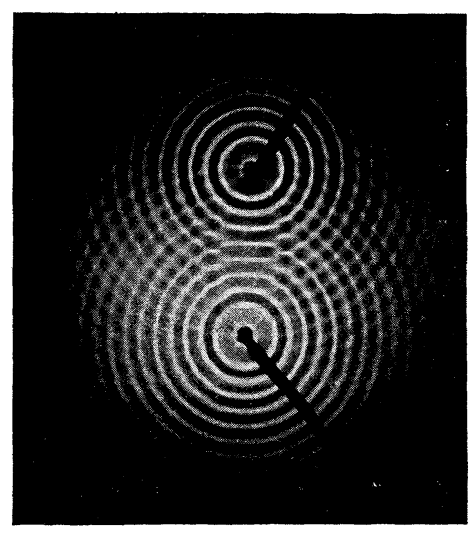

Fig. 3.

Interference of two sets of circular waves.

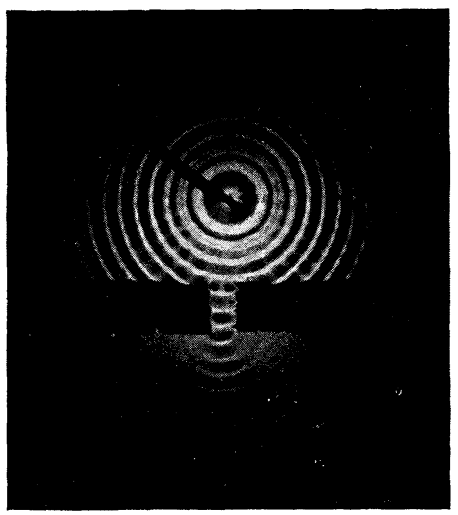

Fig. 5.

Diffraction through a narrow channel. Also reflection of waves.

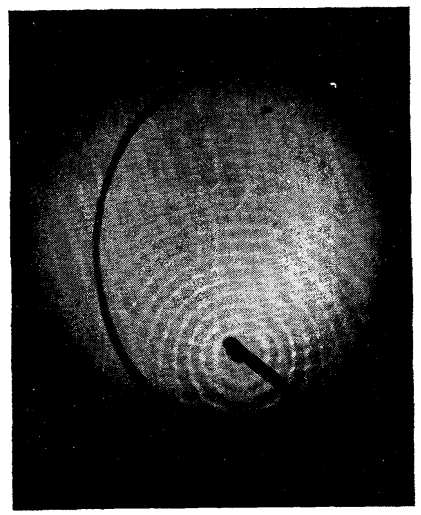

Fig. 7.

Reflection of waves in ellipse. Note the conjugate focus.

F. R. WATSON AND W. A. SHEWART. 


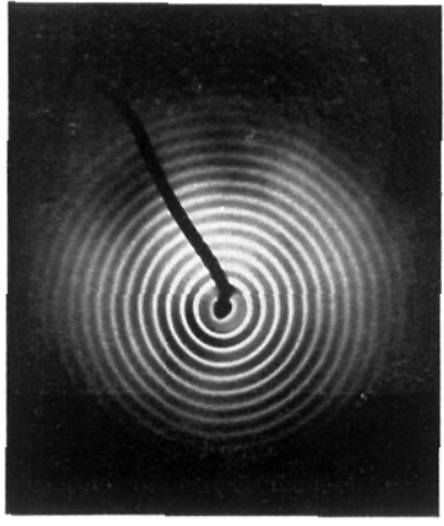

Fig. 2.

Circular waves.

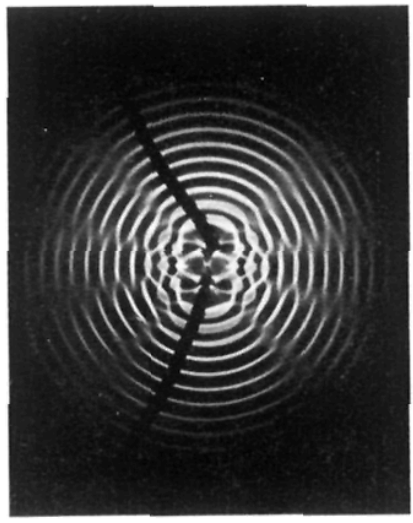

Fig. 4.

Interference of two sets of circular waves with sources close together.

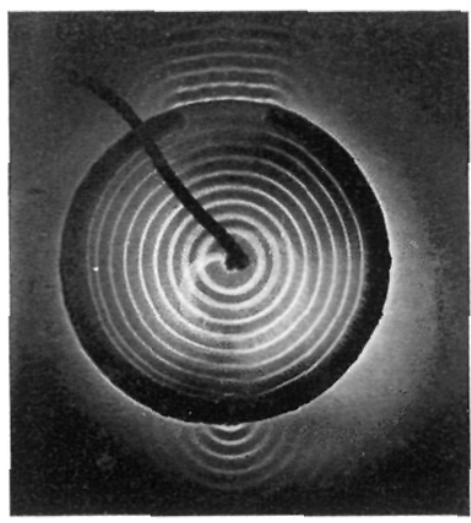

Fig. 6.

Diffraction of waves through narrow and wide aperture.

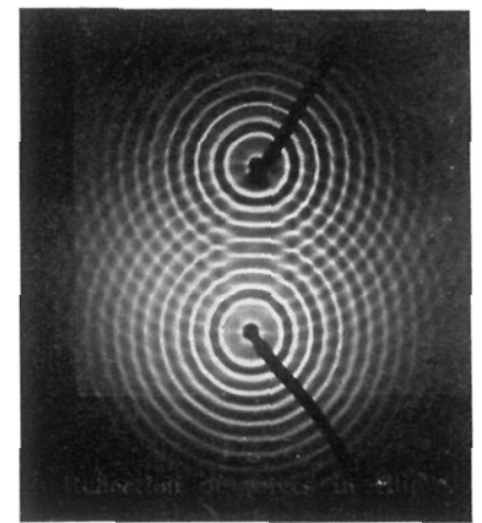

Fig. 3.

Interference of two sets of circular waves.

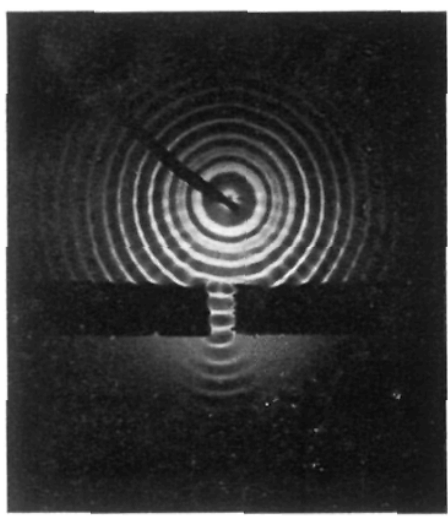

Fig. 5.

Diffraction through a narrow channel. Also reflection of waves.

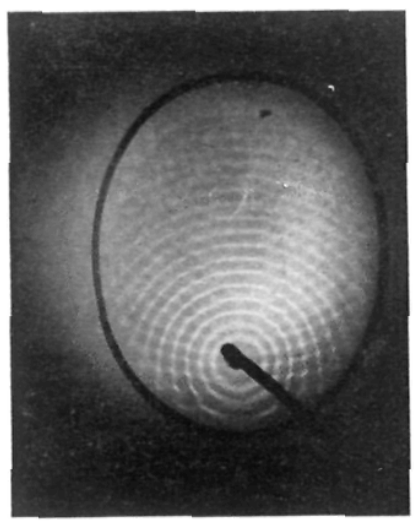

Fig. 7.

Reflection of waves in ellipse. Note the conjugate focus. 